\title{
MECHANICAL SYSTEMS WITH SYMMETRY ON HOMOGENEOUS SPACES
}

\author{
BY \\ ERNESTO A. LACOMBA(1)
}

\begin{abstract}
The geodesic flow on a homogeneous space with an invariant metric can be naturally considered within the framework of Smale's mechanical systems with symmetry. In this way we have at our disposal the whole method of Smale for studying such systems. We prove that certain sets $\Sigma^{\prime}, \Sigma, \operatorname{Im}, \sigma$ and Re which play an important role in the global behavior of those systems, have a particularly simple structure in our case, and we also find some geometrical implications about the geodesics. The results obtained are especially powerful for the case of Lie groups, as in the rigid body problem.
\end{abstract}

1. Introduction. Since Smale developed his theory of mechanical systems with symmetry to study the plane $n$-body problem from a global viewpoint [12], [13], a few applications have been considered to other mechanical systems admitting some symmetry. We study here from the global analysis viewpoint geodesics on homogeneous spaces with a Riemannian metric invariant under the group action, as one more application of Smale's theory.

Roughly speaking, the paper is carried out as follows. The sets $\Sigma^{\prime}, \Sigma, \operatorname{Im}, \sigma$ and $\mathrm{Re}$ associated with any mechanical system with symmetry furnish an important part of its global structure $(\$ 2)$. We show that those sets have particularly simple structure for our problem under consideration, and then we find some geometrical implications about the corresponding geodesics.

The rigid body problem is the most classical example where our general results apply, and Iacob [6] has already studied some aspects of it by using Smale's theory (see end of $\$ 3$ ).

$\S 2$ is devoted to notation and a quick review of Smale's theory. In $\$ 3$ we study properties of structure and invariance under the group action for the five abovementioned sets. By exploiting the transitivity we find that in some precise sense they can be generated from much simpler sets. In particular, that simpler set $\mathbf{R e}^{\circ}$

Received by the editors December 27, 1972.

AMS (MOS) subject classifications (1970). Primary 53C30, 58F05; Secondary 70G05, 70H99, $70 \mathrm{El} 5$.

Key words and phrases. Global viewpoint, mechanical systems with symmetry, rigid body problem, kinetic and potential energy, momentum, invariant submanifolds, bifurcation set, relative equilibria, $G$-invariant metric, algebraic and semialgebraic sets, algebraic manifold, algebraic linear group.

(1) This work contains the results of the author's doctoral thesis at the University of California, Berkeley, and I would like to acknowledge Professor Steve Smale for his helpful advice to this research. I would also like to thank Professor C. Simon and S. Kobayashi for many helpful comments. The research was supported by the Consejo Nacional de Ciencia y Tecnologia and Instituto Mexicano del Petróleo (Mexico City).

Copyright $\odot 1974$, American Mathematical Society 
corresponding to the relative equilibria $\operatorname{Re}$ can be constructed in purely algebraic terms.

In $\S 4$ we study conditions for the sets $\Sigma^{\prime}$, Im, $\sigma$ to be algebraic or semialgebraic. The main result shows that if manifolds and maps are algebraic, then those sets are semialgebraic. These results might be generalized for less restrictive conditions on the manifolds and maps by the transitivity of the group action.

$\$ 5$ considers geometrical properties for geodesics on our transitive mechanical systems. The main result states that any self-intersecting geodesic must be closed (generalizing a result by Kostant), while the second one assures, for $M$ compact, the existence of some unit speed geodesic, all of whose velocity vectors are contained in the relative equilibria $\mathrm{Re}$.

2. Preliminaries. Our main references for basic results and notation will be Smale [12], [13] and Robbin [11]. All maps and manifolds will be assumed to be $C^{\infty}$ differentiable.

If $M$ is a manifold, $\tau_{M}: T M \rightarrow M$ will denote its tangent bundle. For a map between manifolds $f: M \rightarrow N, D f: T M \rightarrow T N$ will denote the derivative of $f$. Also, $\sigma(f), \Sigma^{\prime}(f)$ and $\operatorname{Im}(f)=f(M)$ will denote the sets of critical points, critical values, and image set of $f$, respectively.

The bifurcation set of $f, \Sigma(f)$, is the set of points $y \in \operatorname{Im}(f)$ over which $f$ fails to be locally trivial, i.e., $y \notin \Sigma(f)$ for $y \in \operatorname{Im}(f)$ if there exist an open set $U$ containing $y$ and a map $g: f^{-1}(U) \rightarrow f^{-1}(y)$ such that $h=(f, g): f^{-1}(U) \rightarrow U$ $\times f^{-1}(y)$ is a diffeomorphism. Clearly, $\Sigma^{\prime}(f) \subset \Sigma(f) \subset \operatorname{Im}(f)$.

We remark here that our definition of bifurcation set is slightly different from Smale's. He takes $y \in N$ whenever we considered $y \in \operatorname{Im}(f)$ in the above definition. The difference is not essential, and we win some generality in the statement of our results.

If $G$ is a Lie group, we denote its Lie algebra by the corresponding script letter 9. Denote by exp: $\mathcal{G} \rightarrow G$ the exponential map, and for $g \in G, L_{g}: G \rightarrow G$, $R_{g}: G \rightarrow G$ will be the left and right translations by $g$. Let ad: $G \rightarrow G L(\mathcal{G})$ be the adjoint representation of $G$ on $\mathcal{G}$, inducing a representation $\operatorname{ad}_{*}: G$ $\rightarrow G L\left(G^{*}\right)$ on the vector space dual of the Lie algebra by

$$
\operatorname{ad}(g)_{*} \theta=\theta \circ \operatorname{ad}\left(g^{-1}\right) \text { for } g \in G, \theta \in \mathcal{G}^{*} \text {. }
$$

If $G$ acts on the manifold $M$ by the left, we have in a natural way an induced left action of $G$ on $T M$, which is denoted by $g_{*} X_{a}$, for $g \in G, X_{a} \in T M$. Corresponding to the same action, we will consider for each $Y \in \mathcal{G}$ the induced Killing vector field $\mathrm{Y}^{+}$on $\boldsymbol{M}$.

A mechanical system with symmetry is a quadruple $(M, K, V, G)$ where $M$ is a manifold (configuration space, the tangent bundle $T M$ being the phase space). $M$ possesses a Riemann metric $\langle,\rangle_{K}$ and $K$ is the square of the norm of this metric (kinetic energy). $V$ is a map on $M$ (potential energy). Finally, $G$ is a Lie group 
acting on $M$ and leaving $K$ and $V$ invariant. The total energy of the system $E: T M \rightarrow R$ is defined by

$$
E=K+V \circ \tau_{M}
$$

determining in a standard way a vector field $X_{E}$ on $T M$ (the Lagrange vector field), which is a second order equation on $M$ describing the dynamics of the system (Abraham [1, pp. 119-122]).

The momentum of the system is a map $J: T M \rightarrow \mathcal{G}^{*}$ defined by

$$
J\left(X_{a}\right) \cdot Y=\left\langle X_{a}, Y^{+}(a)\right\rangle_{K} \text { for } X_{a} \in T_{a} M, a \in M, Y \in \mathcal{G} \text {. }
$$

Since $E$ and $J$ are first integrals of $X_{E}$, that is, they are constant along integral curves of $X_{E}[12, \S 4]$ then the energy-momentum map, defined as

$$
I=(E, J): T M \rightarrow R \times \mathcal{G}^{*}
$$

will also be a first integral.

The function satisfies the following equivariance property:

$$
I\left(g_{*} X_{a}\right)=\left(E\left(X_{a}\right), \operatorname{ad}(g)_{*} J\left(X_{a}\right)\right)
$$

for $g \in G, X_{a} \in T M$.

Given any $(c, p) \in R \times \mathcal{G}^{*}$, the set $I_{c, p}=I^{-1}(c, p)$ is invariant under the flow of $X_{E}$, since $I$ is a first integral. In fact, for "almost all" values $(c, p) \in R \times \mathcal{G}^{*}$, the sets $I_{c, p}$ are invariant manifolds (possibly empty) for $X_{E}[12$, p. 318].

Because of the above, the topological structure of $I$ permits to understand the global topological picture of a mechanical system with symmetry, as described by Smale [12]. The determination of the sets of bifurcation $\Sigma(I)$ and critical values $\Sigma^{\prime}(I)$ furnishes a very important part of this topological structure, since the first one contains all the points of $\operatorname{Im}(I)$ in whose neighborhood the topology of the invariant "submanifolds" is likely to change, while the second one contains all the points where the $I_{c, p}$ might not really be submanifolds of $T M$.

Recall that $\Sigma^{\prime}(I) \subset \Sigma(I) \subset \operatorname{Im}(I)$. They are all equal if $2 \operatorname{dim} M<\operatorname{dim} G$ +1 , and $\Sigma^{\prime}(I)=\Sigma(I)$ holds for $M$ compact. Because of that, a study of the properties of $\Sigma(I), \Sigma^{\prime}(I)$ must also include a study of the set $\operatorname{Im}(I)$.

From now on the symbols $\sigma, \Sigma^{\prime}, \Sigma$ and Im without any further notice will mean the corresponding sets for $I: T M \rightarrow R \times G^{*}$.

The relative equilibria $\operatorname{Re}$ for our mechanical system is the set of points $X_{a} \in T M$ such that if $\gamma(t)$ is the integral curve of $X_{E}$ through $X_{a}$, then we have $\gamma(t)=(\exp t Y)_{*} X_{a}$, for some $Y \in \mathcal{G}$.

This is the relative equilibria set in the sense of Robbin, while Smale's is the projection $\tau_{M}(\mathrm{Re})$.

The set $\operatorname{Re}$ contains exactly the orbits of $X_{E}$ which can be simply characterized by the action of one-parameter subgroups of $G$. In addition, it is of interest towards the determination of $\Sigma^{\prime}(I)$ because of the following result (Robbin [11, Theorem 7.1]). 
(2.6) Let $\Lambda$ be the set of $a \in M$ such that the isotropy subgroup $G_{a}$ $=\{g \in G \mid g \cdot a=a\}$ has dimension greater than zero. Then

(a) in general $\operatorname{Re} \subset \sigma$;

(b) $\operatorname{Re}=\sigma$, for $\Lambda=\varnothing$.

If $G$ is a Lie group and $H \subset G$ a closed subgroup, then $G / H$ is a homogeneous space with the standard manifold structure. Let $\Pi: G \rightarrow G / H$ denote the canonical projection, with its derivative at the identity $D \Pi: G \rightarrow T_{o}(G / H)$, where $o=e H$.

A transitive mechanical system (with symmetry) is a mechanical system with symmetry, where $M=G / H$ is a homogeneous space of the symmetry group $G$ with the usual transitive action $G \times G / H \rightarrow G / H$, and with potential $V=0$. We will denote it by the triple $S=(G / H, K, G)$ and will say that the associated Riemann metric is $G$-invariant, or simply invariant. The total energy here is $E=K$. In this work we will only consider transitive mechanical systems.

Geometrically speaking, in mechanical systems where the potential function is identically zero the vector field $X_{E}$ will describe exactly the geodesic flow in phase space. Hence, the study of transitive mechanical systems will be just the study of geodesics on homogeneous spaces with invariant metrics now using Smale's methods, and we will be especially concerned with structural and related geometric properties of the sets $\Sigma^{\prime}, \Sigma, \mathrm{Im}, \sigma$ and $\mathrm{Re}$, as already mentioned.

The integral curves $\gamma(t)$ of the Lagrange vector field $X_{E}$ on $T M$ will be called derivatives of geodesics, while their projections will be geodesics on $M=G / H$.

For transitive mechanical systems the condition $\Lambda=\varnothing$ in the result (2.6)(b) should be replaced by the equivalent one, $\operatorname{dim} H=0$.

The following handy property from linear algebra is useful for actual computation of $J$ in examples and for checking the algebraic properties in Corollaries 4.3 and 4.4 .

(2.7) Given a fixed basis $\left\{Y_{1}, \ldots, Y_{n}\right\}$ of $\mathcal{G}$, there is an isomorphism $\mathcal{G}^{*} \rightarrow R^{n}$ defined by sending $\alpha \in \mathcal{G}^{*}$ into $\left(\alpha\left(Y_{1}\right), \ldots, \alpha\left(Y_{n}\right)\right) \in R^{n}$. This induces a representation of the momentum as a map $J: T M \rightarrow R^{n}$, by

$$
J\left(X_{a}\right)=\left(J\left(X_{a}\right) \cdot Y_{1}, \ldots, J\left(X_{a}\right) \cdot Y_{n}\right) .
$$

3. Structure and invariance properties of the sets $\Sigma^{\prime}, \Sigma$, Im, $\sigma$ and Re. Consider a transitive mechanical system $S=(M, K, G)$. In this and the following section, let $\Delta(\cdot)$ denote either $\operatorname{Im}(\cdot), \Sigma(\cdot)$ or $\Sigma^{\prime}(\cdot)$ and $\Gamma$ denote either $\sigma(I)$ or Re.

Observe that the group $G$ acts on $R \times G^{*}$ by $\operatorname{ad}(g) \cdot(c, p)=\left(c, \operatorname{ad}(g)_{*} p\right)$, for $g \in G$ and $(c, p) \in R \times \mathcal{G}^{*}$.

With this notation, equation (2.5) can be written as

$$
\operatorname{ad}(g) \circ I=I \circ g^{*} \text { for any } g \in G \text {. }
$$

We say that $I: T M \rightarrow R \times G^{*}$ is equivariant under the actions of $G$ on $T M$ and on $R \times \mathcal{G}^{*}$. 
Theorem 3.1. The sets $\Delta(I)$ are invariant under the action of $G$ on $R \times G^{*}$ as defined above.

Proof. The result for $\Delta=\operatorname{Im}$ is clear from equation (3.1). Let $\Delta=\Sigma^{\prime}$ now. Taking derivatives from both sides of (3.1) with the help of the chain rule, and recalling that the isomorphisms $\operatorname{ad}(g)$ and $g_{*}$ induce isomorphisms at the derivatives level, we see that $X_{a} \in \sigma(I)$ if and only if $g_{*} X_{a} \in \sigma(I)$ for any $g \in G$. Again by (3.1), this shows that $(c, p) \in \Sigma^{\prime}(I)$ implies $\operatorname{ad}(g) \cdot(c, p)$ $\in \Sigma^{\prime}(I)$. So, $\Sigma^{\prime}$ is $G$-invariant.

In the case $\Delta=\Sigma$, we will show that $\operatorname{Im}(I)-\Sigma(I)$ is $G$-invariant.

Let $(c, p) \in \operatorname{Im}-\Sigma$, that is, there exist an open set $U \subset R \times g^{*}$ containing (c, $p)$, and a differentiable map $h: I^{-1}(U) \rightarrow I^{-1}(c, p)$ such that $(I, h): I^{-1}(U)$ $\rightarrow U \times I^{-1}(c, p)$ is a diffeomorphism.

By using the equivariance equation (3.1), we see that the new map $\left(I, g_{*}^{-1} \circ h\right.$ - $g_{*}$ ) for $g \in G$ is a diffeomorphism, since it can be written as the composition of three diffeomorphisms:

$$
\left(I, g_{*}^{-1} \circ h \circ g_{*}\right)=\left(\operatorname{ad}(g)^{-1}, g_{*}^{-1}\right) \circ(I, h) \circ g_{*} .
$$

This shows that $\operatorname{ad}\left(g^{-1}\right) \cdot(c, p) \in \operatorname{Im}-\Sigma$, as required. Q.E.D.

Recall now that $O=e H$ is the reference point in the homogeneous space $M=G / H$. The superscript $o$ in $\sigma^{o}(I), \operatorname{Re}^{o}$ will denote the intersection of the corresponding sets without superscript with the tangent space $T_{o} M$.

Also, $\operatorname{Im}_{o}(I)$ will denote the image of $T_{o} M$ under $I$, and $\Sigma_{o}^{\prime}(I), \Sigma_{o}(I)$ will mean the intersection of $\operatorname{Im}_{o}(I)$ with the corresponding sets without subscript. The symbols $\sigma^{\circ}, \Sigma_{o}^{\prime}, \Sigma_{o}$ and $\operatorname{Im}_{o}$ without any further notice will be understood to refer to the map $I$.

Moreover, $\Delta_{o}(\cdot)$ and $\Gamma^{o}$ will have the obvious meaning, according to the convention at the beginning of this section.

As a consequence of Theorem 3.1 and the transitivity of the group action for $S$, we get the following result.

Corollary 3.2. In $S$, we have

$$
\begin{aligned}
\Delta(I) & =\left\{\left(c, \operatorname{ad}(g)_{*} p\right) \in R \times \mathcal{G}^{*} \mid(c, p) \in \Delta_{o}(I), g \in G\right\}, \\
\Gamma & =\left\{g_{*} X_{o} \mid g \in G, X_{o} \in \Gamma^{o}\right\} .
\end{aligned}
$$

Proof. There is only one point to prove and it is that $R e$ is invariant under the action of $G$ on $T M$.

Given $X_{a} \in \operatorname{Re}, g \in G$, we are required to prove $g_{*} X_{a} \in \operatorname{Re}$. Since $X_{a} \in \operatorname{Re}$, the integral curve to $X_{E}$ through $X_{a}$ is given by $\gamma(t)=(\exp t Y)_{*} X_{a}(Y \in \mathcal{G})$.

Hence $g_{*} \gamma(t)=\left(g \exp t Y g^{-1}\right)_{*}\left(g_{*} X_{a}\right)$, which shows $g_{*} X_{a} \in \operatorname{Re}$, since $g_{*}$ takes integral curves into integral curves, and $g \exp t \mathrm{Yg}^{-1}$ is the one-parameter subgroup associated to $\operatorname{ad}(g) Y$. Q.E.D. 
Let $T_{1} M$ be the unit tangent bundle of $M$ in the metric $\langle,\rangle_{K}$, and denote by $J^{*}$ the restriction of the momentum function $J$ to $T_{1} M$. We can consider the corresponding sets $\Sigma\left(J^{*}\right), \Sigma^{\prime}\left(J^{*}\right)$ and $\operatorname{Im}\left(J^{*}\right)$. As before, we define sets $\operatorname{Im}_{o}\left(J^{*}\right)$ as the image of the $K$-unit sphere $S_{K}^{o}$ of $T_{o} M$ under the map $J, \sigma^{o}\left(J^{*}\right)$ as the set of critical points of $J^{*}$ lying in $S_{K}^{o}$, and $\Sigma_{o}\left(J^{*}\right), \Sigma_{o}^{\prime}\left(J^{*}\right)$ the intersection with $\operatorname{Im}_{o}\left(J^{*}\right)$ of the corresponding sets without subscript.

The importance of this function $J^{*}$ lies in that it permits the reduction of some questions about the structure of $\Sigma^{\prime}(I), \Sigma(I)$ or $\operatorname{Im}(I)$ to the corresponding questions about $\Sigma^{\prime}\left(J^{*}\right), \Sigma\left(J^{*}\right)$ or $\operatorname{Im}\left(J^{*}\right)$ respectively, as shown by the following result and Theorem 4.1.

Theorem 3.3. For a transitive mechanical system:

(a) The set $\sigma^{\circ}$ is a cone in $T_{o} M$, generated by $\sigma^{\circ}\left(J^{*}\right)$.

(b) The set $\operatorname{Re}^{0}$ is also a cone in $T_{o} M$.

(c)(i), $p \in \Delta\left(J^{*}\right) \Leftrightarrow-p \in \Delta\left(J^{*}\right)$;

(ii) $\Delta(I)=\left\{\left(\lambda^{2}, \lambda p\right) \in R \times \mathcal{G}^{*} \mid p \in \Delta\left(J^{*}\right), \lambda \in R\right\} \cup 0$.

The last part simply says that the sets $\Delta\left(J^{*}\right)$ are symmetric with respect to the origin in $\mathcal{G}^{*}$, and the $\Delta(I)$ are generated by considering the parabolas with axis $R \times 0 \subset R \times Q^{*}$, vertex at the origin and passing through the corresponding $1 \times \Delta\left(J^{*}\right)$.

For this theorem we will need the following lemma, which is obtained in the same spirit as Proposition 6.1 in [12].

Lemma 3.4. Let $X_{a} \in T M$ such that $K\left(X_{a}\right)=c \neq 0$. Then $X_{a}$ is a critical point for $I$ if and only if $X_{a}$ is a critical point for $J: K^{-1}(c) \rightarrow G^{*}$.

Proof of the theorem. (a) We first prove that if $X_{a} \in \sigma(I)$ and $\lambda$ real different from zero, then $\lambda X_{a} \in \sigma(I)$.

Notice that any real $\lambda \neq 0$ induces a diffeomorphism of $T M$ by scalar multiplication on fibers and denoted by the same letter

$$
\lambda: T M \rightarrow T M, \quad \lambda\left(X_{a}\right)=\lambda \cdot X_{a},
$$

with derivative $\lambda_{*}: T(T M) \rightarrow T(T M)$.

Now, $D I\left(X_{a}\right)=\left(D K\left(X_{a}\right), D J\left(X_{a}\right)\right)$ and we can easily check that

$$
D I\left(\lambda X_{a}\right)=\lambda\left(\lambda D K\left(X_{a}\right), D J\left(X_{a}\right)\right) \circ \lambda_{\neq}^{-1} \text { for } \lambda \neq 0 .
$$

This shows from linear algebra that if $\operatorname{DI}\left(X_{a}\right)$ is not onto, then $\operatorname{DI}\left(\lambda X_{a}\right)$ is not onto either, as required.

Finally, for a given $X_{a} \in T M, X_{a} \in \sigma(I)$ if and only if $X_{a} \in \sigma\left(J^{*}\right)$. This follows from the lemma for $c=1$.

(b) We prove that if $X_{a} \in \operatorname{Re}$ and $\lambda$ real different from zero, then $\lambda X_{a} \in \operatorname{Re}$. From the definition we see that $X_{a} \in \operatorname{Re}$ means that $\gamma(t)=(\exp t Y)_{*} X_{a}$ for 
some $Y \in \mathcal{G}$ is the integral curve of $X_{E}$ through $X_{a}$. Then $\lambda \gamma(t)=(\exp t Y)_{*} \lambda X_{a}$ shows that $\lambda X_{a} \in \mathrm{Re}$, as required.

(c) The result for $\Delta=\mathrm{Im}$ is clear since

$$
I\left(\lambda X_{a}\right)=\left(\lambda^{2}, \lambda J^{*}\left(X_{a}\right)\right) \text { for } X_{a} \in T_{1} M \text { and } \lambda \text { real . }
$$

The case $\Delta=\Sigma^{\prime}$ follows from part (a) and formula (3.3) again, since the set of critical values of any map is the image of its set of critical points.

The case $\Delta=\Sigma$ requires a longer reasoning. To get both parts (i) and (ii), it will be enough to show that $p_{o} \notin \Sigma\left(J^{*}\right)$ if and only if $\left(\lambda^{2}, \lambda p_{o}\right) \notin \Sigma(I)$ for any $\lambda \neq 0$.

Assume first $p_{o} \notin \Sigma\left(J^{*}\right)$, and $\lambda \neq 0$ given. This means that there exist an open neighborhood $U \subset \mathcal{G}^{*}$ of $p_{o}$ and a map $h: J^{*-1}(U) \rightarrow J^{*-1}\left(p_{o}\right)$ such that $\left(J^{*}, h\right): J^{*-1}(U) \rightarrow U \times J^{*-1}\left(p_{o}\right)$ is a diffeomorphism.

Let $\alpha, \beta$ be reals such that $0<\alpha<1<\beta$, then the set

$$
U_{\lambda}=\left\{\left(\lambda^{2} t^{2}, \lambda t p\right) \in R \times \mathcal{G}^{*} \mid p \in U, t \in(\alpha, \beta)\right\}
$$

is an open neighborhood of $\left(\lambda^{2}, \lambda p_{o}\right)$, so that

$$
I^{-1}\left(U_{\lambda}\right)=\left\{\lambda t X_{a} \mid X_{a} \in J^{*-1}(U) \subset T_{1} M, t \in(\alpha, \beta)\right\}
$$

is also open. In fact, they are simply related to the corresponding sets for $J^{*}$ by the diffeomorphisms $\psi:(\alpha, \beta) \times U \rightarrow U_{\lambda}$ defined by $\psi(t, p)=\left(\lambda^{2} t^{2}, \lambda t p\right)$, and $\phi:(\alpha, \beta) \times J^{*-1}(U) \rightarrow I^{-1}\left(U_{\lambda}\right)$ defined by $\phi\left(t, X_{a}\right)=\lambda t X_{a}$.

We claim that $h_{o}: I^{-1}\left(U_{\lambda}\right) \rightarrow I^{-1}\left(\lambda^{2}, \lambda p_{o}\right)$, defined by $h_{o}\left(\lambda t X_{a}\right)=h\left(X_{a}\right)$ for $t \in(\alpha, \beta)$ and $X_{a} \in J^{*-1}(U)$, satisfies that $\left(I, h_{o}\right): I^{-1}\left(U_{\lambda}\right) \rightarrow U_{\lambda} \times I^{-1}\left(\lambda^{2}, \lambda p_{o}\right)$ is a diffeomorphism, which shows $\left(\lambda^{2}, \lambda p_{o}\right) \in \Sigma(I)$. This can be seen by checking that the last map can be split as follows:

$$
\left(I, h_{o}\right)=\left(\psi \times \mathrm{id}_{p_{o}}\right) \circ\left(\mathrm{id}_{\alpha, \beta} \times\left(J^{*}, h\right)\right) \circ \phi^{-1}
$$

where $\mathrm{id}_{p_{o}}$ and $\mathrm{id}_{\alpha, \beta}$ are the identity maps in $J^{*-1}\left(p_{o}\right)$ and $(\alpha, \beta)$ respectively, and the right-hand side is a composition of diffeomorphisms.

For the converse, assume $\left(\lambda^{2}, \lambda p_{o}\right) \notin \Sigma(I)$, where $\lambda \neq 0$, which means that there exist an open neighborhood $U_{\lambda} \subset R \times \mathcal{G}^{*}$ of $\left(\lambda^{2}, \lambda p_{o}\right)$ and a map $h$ : $I^{-1}\left(U_{\lambda}\right) \rightarrow I^{-1}\left(\lambda^{2}, \lambda p_{o}\right)$ such that $(I, h): I^{-1}\left(U_{\lambda}\right) \rightarrow U_{\lambda} \times I^{-1}\left(\lambda^{2}, \lambda p_{o}\right)$ is a diffeomorphism. We may say without loss of generality that $U_{\lambda}$ is of the form (3.4) above, where $U \subset 9^{*}$ open and contains $p_{0}$.

We will first reduce the proof to the case $\lambda=1$. Let $U_{1}$ be the set defined by equation (3.4) for $\lambda=1$. Then $\theta: U_{1} \rightarrow U_{\lambda}$, defined by $\theta(c, p)=\left(\lambda^{2} c, \lambda p\right)$, and $\lambda: I^{-1}\left(U_{1}\right) \rightarrow I^{-1}\left(U_{\lambda}\right)$, defined by the restriction of the map in (3.2), are diffeomorphisms.

The new map $\left(I, \lambda^{-1} \circ h \circ \lambda\right): I^{-1}\left(U_{1}\right) \rightarrow U_{1} \times I^{-1}\left(1, p_{o}\right)$ is a diffeomorphism since it can be split as the composition of three diffeomorphisms: 


$$
\left(I, \lambda^{-1} \circ h \circ \lambda\right)=\left(\lambda^{-1} \times \theta\right) \circ(I, h) \circ \lambda .
$$

This shows that $\left(1, p_{o}\right) \notin \Sigma(I)$.

Finally, $(1 \times U) \times I_{1, p_{0}}$ is a submanifold of $U_{1} \times I_{1, p_{0}}$ and the restriction of $\left(I, \lambda^{-1} \circ h \circ \lambda\right)$ to $I^{-1}(1 \times U)=J^{*-1}(U)$ defines a diffeomorphism $\left(J^{*}, \lambda^{-1} \circ h\right.$ $\circ \lambda): J^{*-1}(U) \rightarrow U \times I_{1, p_{o}}=U \times J^{*-1}\left(p_{o}\right)$. This shows that $p_{o} \notin \Sigma\left(J^{*}\right)$. Q.E.D.

Corollary 3.5. In any transitive mechanical system we have $\Delta_{o}(I)=\left\{\left(\lambda^{2}, \lambda p\right)\right.$ $\left.\in R \times G^{*} \mid p \in \Delta_{o}\left(J^{*}\right), \lambda \in R\right\} \cup 0$.

We can describe a method to generate $\Delta$ from the much simpler corresponding set $\Delta_{o}\left(J^{*}\right)$ in two steps consisting of first applying Corollary 3.5 and then Corollary 3.2.

The following proposition shows that under suitable conditions like compactness of $M$ the projection of the set $\operatorname{Im}(I)$ on an appropriate 2-dimensional subspace of $R \times 9^{*}$ is the closed convex region bounded by a parabola (maybe degenerate).

Proposition 3.6. Consider $S=(M=G / H, K, G)$ where $M$ is compact connected with dimension greater than 1.

Let $\mathcal{L} \subset \mathcal{G}$ be a one dimensional Lie subalgebra, proj: $\mathcal{G}^{*} \rightarrow \mathcal{L}^{*}$ being the dual of the corresponding inclusion. Then the energy preserving projection $\mathrm{id}_{R} \times$ proj: $R \times \mathcal{G}^{*} \rightarrow R \times \mathcal{L}^{*}$ takes $\operatorname{Im}(I)$ into a set of the form $\left\{(c, p) \in R \times R \mid \lambda c \geq p^{2}, c\right.$ $\geq 0\}$, where $\lambda \geq 0$ constant.

Proof. We remark that because of the assumptions on $M$, the unit tangent bundle $T_{1} M=K^{-1}(1)$ is also compact connected. By continuity, $\operatorname{Im}\left(J^{*}\right)$ is a compact connected subset of $\mathcal{G}^{*}$.

Now, let $L \subset G$ be the one parameter subgroup corresponding to $\mathcal{L}$. Consider the inclusion $i$ : $\mathcal{L} \rightarrow \mathcal{G}$, its dual proj $=i^{*}$ and the energy preserving projection $\mathrm{id}_{R} \times$ proj: $R \times \mathcal{G}^{*} \rightarrow R \times \mathcal{L}^{*}$.

Let us denote by $J, I=(K, J)$ the maps associated with our mechanical system $S=(M, K, G)$, and by $\bar{J}, \bar{I}=(K, \bar{J})$ the maps associated with the general mechanical system with symmetry $(M, K, 0, L)$ obtained by just restricting the original action of $G$ on $M$ to an action of $L$ on $M$.

It is easy to check that $\mathrm{id}_{R} \times \operatorname{proj}(\operatorname{Im}(I))=\operatorname{Im}(\bar{I})$. This reduces the proof to show that $\operatorname{Im}(\bar{I})$ has the required shape.

Finally, looking at the derived mechanical system, it is clear that since $\mathcal{L}^{*}$ is one dimensional, $\operatorname{Im}\left(\bar{J}^{*}\right)$ is a compact connected subset of the reals, and by symmetry it must be a closed interval of the form $\operatorname{Im}\left(J^{*}\right)=[-\lambda, \lambda]$, where $\lambda \geq 0$. From Theorem 3.3, we get the conclusion. Q.E.D.

Our next goal is to get an explicit description of $\mathrm{Re}^{o}$ as a cone in $T_{o} M$ (Theorem 3.3(b)), giving direct information in some cases about $\sigma^{\circ}(I)$ (see (2.6)), and indirectly about $\Sigma_{o}^{\prime}(I)$. 
In this section we denote by $\nabla$ an affine connection on the homogeneous space $M=G / H$, as is a standard notation. We will say that $\nabla$ is an invariant affine connection on $M$ if it is invariant under the action of any diffeomorphism $g \in G$, that is

$$
g_{*}\left(\nabla_{X} Y\right)=\nabla_{g_{*} X} g_{*} Y
$$

for given vector fields $X$ and $Y$ on $M$.

Given an invariant Riemann metric $\langle,\rangle_{K}$ on a homogeneous space $M$, the corresponding Riemannian connection $\nabla$ is also invariant. We define a bilinear $\operatorname{map} \alpha: \mathcal{G} \times \mathcal{G} \rightarrow T_{0} M$ by

$$
\alpha(X, Y)=\left(\nabla_{X^{+}} Y^{+}\right)_{0} \text { for } X, Y \in \mathcal{G}
$$

The following theorem shows how the relative equilibria in $T_{o} M$ can be defined by a symmetric bilinear map $U$. In addition it shows how to compute $U$ in a purely algebraic way in terms of the induced inner product $\langle,\rangle_{0}$ at $T_{0} M$, the Lie bracket in $\mathcal{G}$, and the linear map $D \Pi: \mathcal{G} \rightarrow T_{o} M$.

Theorem 3.7. If $M=G / H$ is a homogeneous space with an invariant Riemann metric, then

$$
\operatorname{Re}^{0}=D \Pi\{X \in \mathcal{G} \mid U(X, X)=0\}
$$

where $U: \mathcal{G} \times \mathcal{G} \rightarrow T_{0} M$ is a symmetric bilinear map determined by the condition that for any $X, Y, Z \in \mathcal{G}$,

$$
2\langle U(X, Y), D \Pi(Z)\rangle_{0}=\langle D \Pi(X), D \Pi[Y, Z]\rangle_{0}+\langle D \Pi[X, Z], D \Pi(Y)\rangle_{\circ} .
$$

Proof. We will carry out this proof in two main steps.

Step 1. Since $\langle,\rangle_{K}$ is a Riemann metric on $M$, the corresponding invariant connection $\nabla$ is determined by (Helgason [4, p. 48], or Hermann [5, p. 273])

$$
\begin{aligned}
2\left\langle\nabla_{Z} X, Y\right\rangle= & Z\langle X, Y\rangle-\langle X,[Y, Z]\rangle \\
& -Y\langle X, Z\rangle+\langle[Y, X], Z\rangle+X\langle Y, Z\rangle-\langle Y,[X, Z]\rangle
\end{aligned}
$$

for any vector fields $X, Y, Z$ on $M$ (we omit the subscript $K$ at the metric by simplicity).

Let $X \in \mathcal{G}$ be given and $\gamma_{X}(t)=\exp t X$. By invariance of $\nabla$ we see that $\beta_{X}(t)=\gamma_{X}(t) \cdot 0=\Pi(\exp t X)$ is a geodesic if and only if $\left(\nabla_{X^{+}} X^{+}\right)_{0} \equiv \alpha(X, X)$ $=0$. Since $\operatorname{Re}^{0}$ consists exactly of the initial velocity vectors $\beta_{X}^{\prime}(0)=D \Pi(X)$ of geodesics as above, we conclude that $\operatorname{Re}^{0}=D \Pi\{X \in \mathcal{G} \mid \alpha(X, X)=0\}$.

Step 2 . We finally claim that

$$
U(Z, X)=\alpha(Z, X)-\frac{1}{2} D \Pi[Z, X]
$$


is a symmetric bilinear map. Formula 25.2 in [5, p. 354] shows that

$$
X^{+}\left\langle Y^{+}, Z^{+}\right\rangle=\left\langle\left[X^{+}, Y^{+}\right], Z^{+}\right\rangle+\left\langle Y^{+},\left[X^{+}, Z^{+}\right]\right\rangle
$$

for any $X, Y, Z \in \mathcal{G}$. Permuting the letters we get two additional formulas. Substituting the three of them into (3.9) and cancelling terms, we arrive at

$$
2\left\langle\nabla_{Z^{+}} X^{+}, Y^{+}\right\rangle=\left\langle\left[Z^{+}, X^{+}\right], Y^{+}\right\rangle+\left\langle\left[Z^{+}, Y^{+}\right], X^{+}\right\rangle+\left\langle\left[X^{+}, Y^{+}\right], Z^{+}\right\rangle
$$

for any $X, Y, Z \in G$.

Evaluating (3.12) at $o \in M$, and keeping in mind that $\left[X^{+}, Y^{+}\right]=[X, Y]^{+}$and $X^{+}(0)=D \Pi(X)$, we get

$$
2\langle\alpha(Z, X), D \Pi(Y)\rangle_{o}=\langle D \Pi[Z, X], D \Pi(Y)\rangle_{0}+2\langle U(Z, X), D \Pi(Y)\rangle_{o},
$$

where $U(Z, X)$ is defined by condition (3.8).

Since (3.13) is true for any choice of $X, Y, Z \in \mathcal{G}$, it implies (3.10), as required.

If $\operatorname{dim} H=0$, we can identify $T_{o} M=9$ and omit $D \Pi$ from the notation in the theorem. In particular, $\sigma^{\circ}=\operatorname{Re}^{o}$ becomes an algebraic cone in $T_{o} M$.

This theorem is essentially an improvement and generalization of results in Nomizu [10, pp. 50-53] and Arnold [3, p. 338], with their notation meaning slightly different concepts (see [8, pp. 50-52] for a complete discussion).

Recall that a Riemannian homogeneous space is said to be reductive if the following condition is satisfied: There exists a vector subspace $M$ of $\mathcal{G}$ such that $G$ is the direct sum of $M$ and the Lie subalgebra of of $H$, and $\operatorname{ad}(H) \Delta M=\varnothing M$.

A Riemannian symmetric space is a connected Riemann manifold $M$ such that for each $x \in M$ there is an isometry $S_{x}$ of $M$ leaving $x$ invariant and reversing geodesics through $x$, that is, if $\gamma$ geodesic and $\gamma(0)=x$, then $S_{x}(\gamma(t))=\gamma(-t)$. Any Riemannian symmetric space is reductive homogeneous, with a canonical decomposition (see [10, p. 53]).

The following immediate corollaries of Theorem 3.7 and (2.6)(a) show fairly general cases where our parameters under consideration become particularly simple (they are essentially Theorems 13.1 and 13.2 in Nomizu [10]).

Corollary 3.8. If $G / H$ is a reductive homogeneous space with fixed decomposition of its Lie algebra $9=\propto M+\alpha, \operatorname{ad}(H) \propto M=\propto M$ and invariant Riemann metric, satisfying $\left\langle X,[Y, Z]_{D_{M}}\right\rangle+\left\langle[Y, X]_{{ }_{M}}, Z\right\rangle=0$ for any $X, Y, Z \in \mathcal{Z}$, where $\langle$, is the inner product on $M$ induced by its isomorphism with $T_{o} M$, and the subscript in the Lie bracket means to take the oM-component, then

$$
\operatorname{Re}=\sigma=T M \text { and } \Sigma^{\prime}=\Sigma=I m
$$


This corollary contains the case of a Riemannian symmetric space, if we take its canonical decomposition as a reductive space. The fact that $\operatorname{Re}=T M$ here means that all the geodesics are generated by one parameter subgroups, which is well known ([4, p. 173, Theorem 3.3], [10, p. 53, Theorem 15.1]).

Corollary 3.9. Let $G$ be a compact connected Lie group and $H$ a closed subgroup, so that the Lie algebra $\mathcal{G}$ admits an $\operatorname{ad}(G)$-invariant inner product $\langle$,$\rangle . Let 0 M$ be the orthogonal subspace to the subalgebra \& of $\mathrm{H}$, and consider the Riemann metric on $M=G / H$, determined by the restriction of the inner prodict to $M \times \propto M$. Then $\operatorname{Re}=\sigma=T M$ and $\Sigma^{\prime}=\Sigma=\operatorname{Im}$ too, but in addition, Im is contained in the paraboloid $\left\{(c, p) \mid\langle p, p\rangle^{*}=c\right\}$. $(\langle$,$\rangle in G$ defines in a standard way an isomorphism $i: \mathcal{G} \rightarrow \mathcal{G}^{*}$. Then $\langle,\rangle^{*}$ is the unique inner product such that $i$ is an isometry.)

If $H=e$ in addition to the hypothesis of this corollary, then we are considering the case of a Lie group $M=G$ with a bi-invariant metric, and $\operatorname{Im}(I)=\operatorname{Im}_{o}(I)$ is actually the paraboloid described with brackets.

(3.14) Example. Rigid body problem. The motion of a rigid body about a fixed point with no external forces can be described by the geodesics of the Lie group $G=S O(3)$ of rotations in $R^{3}$ acting on itself, with a left invariant metric. This metric is generated by an inner product at the identity whose matrix $A$ corresponds classically to the inertia tensor of the rigid body.

There is a natural isomorphism of the Lie algebra of $S O(3)$ with $R^{3}$, under which the adjoint representation can be considered as the identity, that is $\operatorname{ad}(B)=B$, for any $B \in S O(3)$. Hence the action of $G$ on $R \times G^{*}$ is by rotations on the second factor, and identity on the first factor.

By using Theorem 3.7 with the remark that $\operatorname{Re}^{\circ}=\sigma^{\circ}$ here, we find that this set is the union of eigenspaces for the matrix A. Applying Corollaries 3.5 and 3.2, we get

$$
\begin{aligned}
\operatorname{Im} & =\left\{(c, p) \in R \times\left. R^{3}\left|c \lambda_{1} \leq\right| p\right|^{2} \leq c \lambda_{3}\right\}, \\
\Sigma & =\Sigma^{\prime}=\left\{(c, p) \in R \times\left. R^{3}\left|c \lambda_{i}=\right| p\right|^{2}, i=1,2,3\right\},
\end{aligned}
$$

where $\lambda_{1} \leq \lambda_{2} \leq \lambda_{3}$ are the eigenvalues of $A$ (see [8, pp. 25-29, 58-60] for the detailed analysis). These results coincide with Iacob's [6, p. 1512].

4. Conditions for $\Sigma^{\prime}$, Im, $\sigma$, to be algebraic or semialgebraic. In the classical examples of mechanical systems with symmetry, the sets $\Sigma^{\prime}, \Sigma$ and Im are semialgebraic. However, that seems to depend strongly on the algebraicness of maps and manifolds, and we will investigate this aspect for transitive mechanical systems. As in $\$ 3, \Delta$ denotes $\Sigma^{\prime}, \Sigma$ or Im.

Recall that an algebraic set $V$ and a semialgebraic set $S$ in $R^{n}$ are subsets of the form (for some applications we can assume without loss of generality that $k=1)$ : 


$$
\begin{aligned}
& V=\left\{X \in R^{n} \mid Q_{1}(X)=\cdots=Q_{k}(X)=0\right\}, \\
& S=\left\{X \in R^{n} \mid P_{1}(a, X)=\cdots=P_{k}(a, X)=0 \text { for some } a \in R^{m}\right\},
\end{aligned}
$$

where the $Q_{i}$ are polynomials in $n$ variables and the $P_{i}$ are polynomials in $m+n$ variables. An algebraic map is a function $f: R^{n} \rightarrow R^{m}$ where each coordinate function is a polynomial in $n$-variables (Milnor [9], Abraham-Robbin [2]). It is a rational map, if the coordinate functions are rather quotients of polynomials (defined only where the denominators are not zero).

The following theorem shows that $\Sigma(I), \Sigma^{\prime}(I)$ or $\operatorname{Im}(I)$ is semialgebraic if and only if the respective set for $J^{*}$ is, and except for a suitable modification this correspondence holds also true when we replace semialgebraic sets by algebraic sets (it relies on Theorem 3.3).

Theorem 4.1. For a transitive mechanical system:

(a) $\Delta\left(J^{*}\right)$ is a semialgebraic set if and only if $\Delta(I)$ is a semialgebraic set.

(b) $\Delta\left(J^{*}\right)$ is an algebraic set if and only if $\Delta(I)$ is the intersection of an algebraic set with $\left\{(c, p) \in R \times \mathcal{G}^{*} \mid c \geq 0\right.$ and $\left.c=0 \Leftrightarrow p=0\right\}$.

Proof. Recall that Theorem 3.3(c) states that $\Delta\left(J^{*}\right)$ is symmetric with respect to the origin and

$$
\Delta(I)=\left\{\left(\lambda^{2}, \lambda p\right) \mid \lambda \in R, p \in \Delta\left(J^{*}\right)\right\} \cup 0 .
$$

Part (a) of our theorem is clear from this equation.

However, part (b) requires a more careful proof. If $\Delta(I)$ is an algebraic set, then clearly $\Delta\left(J^{*}\right)$ is an algebraic set, since $1 \times \Delta\left(J^{*}\right)$ is the intersection of $\Delta(I)$ with the hyperplane $c=1$ in $R \times Q^{*}$.

Suppose now

$$
\Delta\left(J^{*}\right)=\left\{p \in \mathcal{G}^{*} \mid Q(p)=0\right\}
$$

is an algebraic set, where $Q$ is polynomial. Then

$$
\Delta(I)=\left\{\left(\lambda^{2}, \lambda p\right) \mid \lambda \in R, p \in \mathcal{G}^{*} \ni Q(p)=0\right\} \cup 0,
$$

and the claim is that there is an algebraic set $W$ in $R \times G^{*}$ such that $\Delta(I)=W \cap\{(c, p) \mid c \geq 0$, and $c=0 \Leftrightarrow p=0\}$.

Since $\Delta\left(J^{*}\right)$ is symmetric with respect to the origin, we may assume without loss of generality that $Q(p)$ in formulas (4.2) and (4.3) contains only terms of either odd or even degree. This is because an arbitrary polynomial can be split into a sum of two such polynomials, inducing a corresponding splitting (in terms of intersection) in (4.2) and (4.3).

For $\lambda \in R, \lambda \neq 0$ and $p \in \mathcal{G}^{*}$, write $\bar{c}=\lambda^{2}, \bar{p}=\lambda p$. Since $Q(p)$ contains only terms of either odd or even degree, the polynomial $\lambda^{\operatorname{deg}(Q)} Q(p)$ can be written as a polynomial $R(\bar{c}, \bar{p})$ such that $R(0,0)=0$, and $Q(p)=0 \Leftrightarrow R\left(\lambda^{2}, \lambda p\right)$ 
$=0$ for all $\lambda \neq 0$. Therefore, $\Delta(I)=\left\{(\bar{c}, \bar{p}) \in R \times \mathcal{G}^{*} \mid \bar{c}>0, R(\bar{c}, \bar{p})=0\right\}$ $\cup 0$, from which the assertion follows with $W=\{(c, p) \mid R(c, p)=0\}$. Q.E.D.

By an algebraic manifold, we will understand an algebraic set $V \subset R^{n}$ which is the inverse image of a regular value of an algebraic map $g: R^{n} \rightarrow R^{k}$. If $V \subset R^{n}$ is an algebraic manifold, then $T V \subset R^{n} \times R^{n}$ is also an algebraic manifold. A Riemann metric $\langle,\rangle_{K}$ on $V$ will be said to be algebraic if the associated kinetic energy $K: T V \rightarrow R$ is an algebraic map.

A linear group $G$ will be a Lie subgroup of $G L(n)(n \times n$ nonsingular real matrices). If in addition $G$ is an algebraic manifold, we will call it an algebraic linear group.

Let $\nabla$ denote now the gradient for real valued maps of several variables.

Theorem 4.2. If $S=(G / H, K, G)$ is a transitive mechanical system where $M=G / H$ is an algebraic manifold and $I$ is a rational function everywhere defined on $T M$, then $\sigma, \Sigma^{\prime}$ and $\operatorname{Im}$ are semialgebraic sets.

Proof. Assume that the algebraic manifold $V=T M$ in $R^{n}$ can be represented by $Q_{1}\left(X_{1}, \ldots, X_{n}\right)=0, \ldots, Q_{k}\left(X_{1}, \ldots, X_{n}\right)=0$, where $\operatorname{dim} V=n-k$ and $Q_{1}$, $\ldots, Q_{k}$ linearly independent on $V$ (by definition).

We will first prove that $\sigma(I)$ is a semialgebraic set. Let $I=\left(I_{1}, \ldots, I_{m}\right)$, where the $I_{i}$ are rational functions into $R$. By a generalization of Lagrange multipliers method, $a \in V$ is a critical point of $I$ if and only if $\nabla Q_{1}(a), \ldots, \nabla Q_{k}(a), \nabla f_{1}(a)$, $\ldots, \nabla f_{m}(a)$ are linearly independent, that is

$$
\lambda_{1} \nabla Q_{1}(a)+\cdots+\lambda_{k} \nabla Q_{k}(a)+\lambda_{k+1} \nabla f_{1}(a)+\cdots+\lambda_{k+m} \nabla f_{m}(a)=0
$$

for $\left(\lambda_{1}, \ldots, \lambda_{k+m}\right) \neq(0, \ldots, 0)$. Therefore, $\sigma(I)=\{a \in V \mid(4.4)$ is satisfied with $\left.\left(\lambda_{1}, \ldots, \lambda_{k+m}\right) \in R^{k+m}, \lambda_{1}^{2}+\cdots+\lambda_{k+m}^{2}>0\right\}$ is a semialgebraic set, since (4.4) can be rewritten as $n$ polynomial equations equated to zero.

Finally, $\Sigma^{\prime}$ and $\mathrm{Im}$ are semialgebraic too, for being the image under the rational map $I$ of the semialgebraic sets $\sigma$ and $V$, respectively. Q.E.D.

The following immediate corollaries of Theorem 4.2 show more concrete cases where manifolds are algebraic, maps are rational, and the actions of the group are defined as restriction of the linear action on $R^{n}$ or as group multiplication.

Corollary 4.3. Let $G \subset G L(n)$ be an algebraic linear group, and $H \subset G$ a closed subgroup such that $M=G / H$ is an algebraic manifold in $R^{n}$ with $G$ acting on $M$ by linear action the obvious way. Assume in addition that we have any G-invariant algebraic Riemann metric on $M$. Then, $\sigma, \Sigma^{\prime}$ and $\operatorname{Im}$ are semialgebraic sets.

Corollary 4.4. Let $G \subset G L(n)$ be an algebraic linear group with a left invariant Riemann metric. Then $\sigma, \Sigma^{\prime}$ and $\operatorname{Im}$ are semialgebraic sets.

5. Geometric properties of geodesics. In this section we study more concrete geometrical properties for geodesics on homogeneous spaces with an invariant 
metric, considered as transitive mechanical systems. The first result is an interesting generalization of Kostant's theorem [7, p. 260].

Theorem 5.1. On a homogeneous space $G / H$ with an invariant metric, any selfintersecting geodesic must be periodic.

Proof. Consider $S=(G / H, K, G)$ as a transitive mechanical system; then $J: T M \rightarrow \mathcal{G}^{*}$ is always linear injective on fibers. Therefore, for any $p \in \mathcal{G}^{*}$ the corresponding set $J_{p}=J^{-1}(p)$ contains at most one point on each fiber of $T M$. The same property holds for the set $I_{c, p}=I^{-1}(c, p)$ for given $(c, p) \in R \times \mathcal{G}^{*}$, since $I_{c, p} \subset J_{p}$.

Therefore, given any geodesic $\gamma(t)$, its derivative $\gamma^{\prime}(t)$ must be contained in some $I_{c, p}$, and by the above property $\gamma^{\prime}(t)$ contains at most one point in $T_{a} M$, for any $a \in M$. So, the conclusion is clear. Q.E.D.

From here it is plausible that geodesics in transitive mechanical systems have a better chance of being closed than geodesics in an arbitrary Riemann manifold, since they cannot have self-intersections without being closed.

Proposition 5.2. On a transitive mechanical system with $M=G / H$ compact, there is at least one unit speed geodesic of $M$ generated by a one parameter subgroup of $G$.

Proof. From Theorem 4.1 in [11], $X_{a} \in \operatorname{Re}$ if and only if there exists $Y \in \mathcal{G}$ such that $X_{a}=Y^{+}(a)$ and $a \in M$ is a critical point for the map $L_{Y}=K \circ Y^{+}$: $M \rightarrow R$.

Since any smooth map on a compact manifold has a critical point, this means that there is at least one unit speed geodesic of the form $\gamma(t)=\lambda \exp t Y \cdot a(\lambda$ real, $a \in M$ ), for any $Y \in \mathcal{G}$ such that $Y^{+}$is not trivial. Q.E.D.

This section might lead to further research in the direction of generic properties of geodesics in transitive mechanical systems.

\section{BIBLIOGRAPHY}

1. R. Abraham, Foundations of mechanics, $A$ mathematical exposition of classical mechanics with an introduction to the qualitative theory of dynamical systems and applications to the three-body problem, Benjamin, New York, 1967. MR 36 \#3527.

2. R. Abraham and J. Robbin, Transversal mappings and flows, Benjamin, New York, 1967. MR 39 \#2181.

3. V.I. Arnol'd, Sur la géométrie différentielle des groupes de Lie de dimension infinie et ses applications a l'hydrodynamique des fluides parfaits, Ann. Inst. Fourier (Grenoble) 16 (1966), fasc. 1, 319-361. MR $34 \# 1956$.

4. S. Helgason, Differential geometry and symmetric spaces, Pure and Appl. Math., vol. 12, Academic Press, New York, 1962. MR 26 \#2986.

5. R. Hermann, Differential geometry and the calculus of variations, Math. in Science and Engineering, vol. 49, Academic Press, New York, 1968. MR 38 \# 1635.

6. A. Iacob, Invariant manifolds in the motion of a rigid body, Rev. Roumaine Math. Pures Appl. 16 (1971), 1497-1521.

7. B. Kostant, On differential geometry and homogeneous spaces. I,II, Proc. Nat. Acad. Sci. U.S.A. 42 (1956), 258-261, 354-357. MR 19, 454. 
8. E. Lacomba, Ph.D. Thesis, University of California, Berkeley, Calif., 1972.

9. J. Milnor, Singular points of complex hypersurfaces, Ann. of Math. Studies, no. 61, Princeton Univ. Press, Princeton, N.J.; Univ. of Tokyo Press, Tokyo, 1968. MR 39 \#969.

10. K. Nomizu, Invariant affine connections on homogeneous spaces, Amer. J. Math. 76 (1954), 33-65. MR 15, 468.

11. J. Robbin, Relative equilibria in mechanical systems, Proc. Internat. Conf. on Dynamical Systems, Bahia, Brazil, 1971.

12. S. Smale, Topology and mechanics. I, Invent. Math. 10 (1970), 305-331.

13._, Topology and mechanics. II, Invent. Math. 11 (1970), 45-64.

Departamento de Matemática, Universidade de Brasília, 70.000-Brasília-DF, Brasil

Current address: C.I.M.A.S., Universidad Nacional Autónoma de México, Apdo. Postal 20-726, México 20, D.F., México 